\title{
Co-doped ZnO nanopowders: Location of cobalt and reduction in photocatalytic activity
}

\author{
Rongliang $\mathrm{He}^{\mathrm{a}}$, Rosalie K. Hocking ${ }^{\mathrm{b}}$, Takuya Tsuzuki ${ }^{\mathrm{a}, *}$ \\ a Centre for Material and Fibre Innovation, Institute for Technology Research and Innovation, Deakin University, Geelong Technology Precinct, Geelong, Victoria, 3217 Australia \\ ${ }^{\mathrm{b}}$ Monash Centre for Synchrotron Science, Australian Centre for Electromaterials Science and School of Chemistry, Monash University, Victoria, 3800 Australia
}

\section{A R T I C L E I N F O}

\section{Article history:}

Received 8 August 2011

Accepted 21 December 2011

\section{Keywords:}

Nanostructures

Sol-gel growth

XAFS (EXAFS and XANES)

Co-doped $\mathrm{ZnO}$

EXAFS

Photocatalysis

\begin{abstract}
A B S T R A C T
The location of dopant ions and the effect of doping level on the photocatalytic activity have been investigated on Co-doped ZnO nanopowders. A co-precipitation method was used to prepare ZnO nanoparticles of $<50 \mathrm{~nm}$ in diameter doped with up to 5 at\% of Co. The crystal structure of nanoparticles and local atomic arrangements around dopant ions were analysed by X-ray diffraction and extended X-ray absorption fine structure spectroscopy using synchrotron radiation. The results showed the Co ions substituted the $\mathrm{Zn}$ ions in $\mathrm{ZnO}$ crystal lattice and induced lattice shrinkage. The photocatalytic activity under simulated sunlight irradiation was characterised by the decomposition of Rhodamine B dye molecules, which revealed the successful reduction of photocatalytic activity by Co-doping.
\end{abstract}

(c) 2012 Elsevier B.V. All rights reserved.

\section{Introduction}

Zinc oxide is an important semiconducting material having a broad range of applications including transparent conductive oxides [1], ultraviolet (UV) light blockers [2] and photocatalysts [3]. ZnO has a bandgap energy of $\sim 3.3 \mathrm{eV}$ that corresponds to the wavelengths of $\sim 375 \mathrm{~nm}$. UV rays with a shorter wavelength than $375 \mathrm{~nm}$ are absorbed by $\mathrm{ZnO}$. $\mathrm{ZnO}$ has been regarded as an excellent UV shielding material with a broad UV absorption characteristics and photo-fastness compared with other organic and inorganic UV shielding materials [4]. However, the inherent photocatalytic activity of ZnO hinders many practical UV-shielding applications [5]. The absorbed UV rays excite the valence electrons onto conduction bands and the photo-generated electrons and holes can move to particle surface to react with electron donors or acceptors to form reactive free radicals such as superoxide $\left(\mathrm{O}_{2}{ }^{-}\right)$and hydroxyl $\left(\bullet^{\circ} \mathrm{OH}\right)$ [6], which causes photocatalysis. Although photocatalysis is of great use in many applications such as water-splitting, organic pollutant scavenging and anti-fouling [7,8], photocatalysis is a serious disadvantage in the use of $\mathrm{ZnO}$ as a safe and durable UV shielding agent, as it can cause skin damage, deterioration of paints and plastics and colour fading of paints and fabrics [9]. Hence, for UV protection applications, it is of particular importance to develop the methods to effectively reduce the photocatalytic properties in $\mathrm{ZnO}$ while optimising its UV-absorption properties.

\footnotetext{
* Corresponding author. Tel.: +61 35227 3205; fax: +613 52272847

E-mail address: takuya@deakin.edu.au (T. Tsuzuki).
}

In the past, impurity doping in semiconductor nanoparticles was investigated mainly to enhance the photocatalytic activity, particularly in the visible light region. Despite the industrial importance of $\mathrm{ZnO}$ nanoparticles as UV blocking agents, the experiments involving the reduction of the photocatalysis of $\mathrm{ZnO}$ nanoparticles have scarcely been reported. Impurity doping may create chemical and, in some cases, physical defects in the crystal lattice that would act as trapping sites of the excitons to reduce the photocatalytic properties $[10,11]$. In this study, the effect of impurity doping on the reduction of photocatalysis in $\mathrm{ZnO}$ was investigated using cobalt as a dopant. Cobalt was selected for its expected ease in doping due to the similar ionic radius $(0.058 \mathrm{~nm})$ to that of $\mathrm{Zn}(0.060 \mathrm{~nm})$. In the past, Co-doping in $\mathrm{ZnO}$ was investigated in order to increase the photocatalytic activity under the visible light $[12,13]$. Dodd et al. produced Co-doped $\mathrm{ZnO}$ nanoparticles by mechanochemical processing and found that the doped $\mathrm{ZnO}$ had reduced photoactivity under the irradiation of monochromised $300 \mathrm{~nm}$ UV light [10]. However, those reports lack the data on the photocatalysis under the entire solar spectrum. Casey et al. reported that Co $0.7 \mathrm{wt} \%$ doped $\mathrm{ZnO}$ showed reduced photoactivity, but the effect of doping level and UV light source used were not reported [14]. To the best of our knowledge, the detailed study about the effect of Co doping level on the photocatalysis under the entire solar spectrum has never been reported. In the present study, cobalt-doped $\mathrm{ZnO}$ were prepared using a co-precipitation method. Different amounts of dopants, namely, $0.25,1,2$ and 5 at\% were studied. Higher doping levels were not studied because of the possibility of forming separate cobalt oxide phases in the nanoparticles [15,16]. X-ray absorption spectroscopy (XAS) was carried out to characterise the 
local atomic arrangement and coordination states of Co element in $\mathrm{ZnO}$ lattice. The change in photocatalytic activity was analysed via the decomposition of Rhodamine B dye molecules under simulated sun-light in the presence of nanopowders.

\section{Experimental}

\subsection{Sample preparation}

Co-doped nanoparticulate $\mathrm{ZnO}$ was synthesized using a coprecipitation method and subsequent heat treatment via the following reaction equations,

$$
\begin{aligned}
& (1-x) \mathrm{CoCl}_{2} \cdot 6 \mathrm{H}_{2} \mathrm{O}+x \mathrm{Zn}\left(\mathrm{CH}_{3} \mathrm{COO}\right)_{2} \cdot 2 \mathrm{H}_{2} \mathrm{O}+\mathrm{Na}_{2} \mathrm{CO}_{3} \\
& \quad \rightarrow \mathrm{Zn}_{x} \mathrm{Co}_{1-x} \mathrm{CO}_{3}+2 x \mathrm{Na}\left(\mathrm{CH}_{3} \mathrm{COO}\right)+2(1-x) \mathrm{NaCl}+\mathrm{H}_{2} \mathrm{O}, \\
& \mathrm{Zn}_{x} \mathrm{Co}_{1-x} \mathrm{CO}_{3} \rightarrow \mathrm{Zn}_{x} \mathrm{Co}_{1-x} \mathrm{O}+\mathrm{CO}_{2} .
\end{aligned}
$$

The raw materials were $\mathrm{Zn}\left(\mathrm{CH}_{3} \mathrm{COO}\right)_{2} \cdot 2 \mathrm{H}_{2} \mathrm{O}$ (Aldrich >99.0\%), $\mathrm{Na}_{2} \mathrm{CO}_{3}$ (Aldrich > 99.0\%) and $\mathrm{CoCl}_{2} \cdot 6 \mathrm{H}_{2} \mathrm{O}$ (Aldrich >98.0\%). First, a mixed solution of $\mathrm{Zn}\left(\mathrm{CH}_{3} \mathrm{COO}\right)_{2} \cdot 2 \mathrm{H}_{2} \mathrm{O}$ and $\mathrm{CoCl}_{2} \cdot 6 \mathrm{H}_{2} \mathrm{O}$ was prepared in distilled water. $\mathrm{Na}_{2} \mathrm{CO}_{3}$ aqueous solution was separately prepared. Then, the mixed solution was added into the $\mathrm{Na}_{2} \mathrm{CO}_{3}$ solution drop-wise while stirring with the speed of $500 \mathrm{rpm}$. White precipitate was immediately formed during the mixing of the two solutions. After $1.5 \mathrm{~h}$ of aging, the slurries were washed with distilled water several times using a centrifuge (DuPont, ECONO SPIN SORVALL Instrument) until the salinity of the supernatant became less than $100 \mathrm{ppm}$. The dried samples were then heat-treated at $350{ }^{\circ} \mathrm{C}$ for $1 \mathrm{~h}$ to decompose $\mathrm{Zn}_{x} \mathrm{Co}_{1-x} \mathrm{CO}_{3}$ into $\mathrm{Zn}_{x} \mathrm{Co}_{1-x} \mathrm{O}$.

\subsection{Powder characterisation}

The morphology of the synthesized particles was characterised by transmission electron microscopy (TEM) using a JEOL JEM-2100 microscope with the beam energy of $200 \mathrm{kV}$. TEM specimen was prepared by evaporating a drop of the nanoparticle dispersion on a carbon-coated specimen grid. A Varian Cary 3E spectrophotometer was used to measure the UV-Vis transmittance spectra of the washed $\mathrm{ZnO}$ and doped $\mathrm{ZnO}$ powders at the wavelength range of $200-800 \mathrm{~nm}$. The actual doping levels in the powders were characterised by Inductively Coupled Plasma-Atomic Emission Spectrometry (ICP-AES) using a Varian Liberty II ICP-AES spectrophotometer.

The crystal structure of the nano-powders was characterised by $\mathrm{X}$-ray powder diffraction (XRD) using the X-ray powder diffraction beamline (wavelength $=0.82591 \AA$ ) at Australian Synchrotron. $c$ Axis lattice parameter was calculated by the Rietveld fitting method using Rietica software package [17].

In order to determine the location of dopants in $\mathrm{ZnO}, \mathrm{X}$ ray absorption spectroscopy (XAS) experiments including both extended X-ray absorption fine structure (EXAFS) and X-ray absorption near edge structure (XANES) were conducted. The samples were diluted with boron nitride (BN) powder to obtain optimum X-ray absorption intensity. Co K-edge XAS spectra were recorded on the multiple wiggler XAS beam-line 12 ID at the Australian Synchrotron, in transmittance operational mode 1 [18]. The beam energy was $3.0 \mathrm{GeV}$ and the maximum beam current was $200 \mathrm{~mA}$. The energy scale was calibrated using a Co foil as an internal standard where the calibration energy $7709.0 \mathrm{eV}$, corresponding to the first inflection point of the foil. The Average program [19], was used to average raw data files and exclude channels. EXAFS data analysis was carried out using Ifeffit software package [20]. The XANES spectrum of Co-methylimidazole $\left(\left[\mathrm{Co}(\mathrm{melm})_{2}\right]_{n}\right)$ was used as a reference sample where Co is tetrahedrally coordinated and has an oxidation state of $2+$.

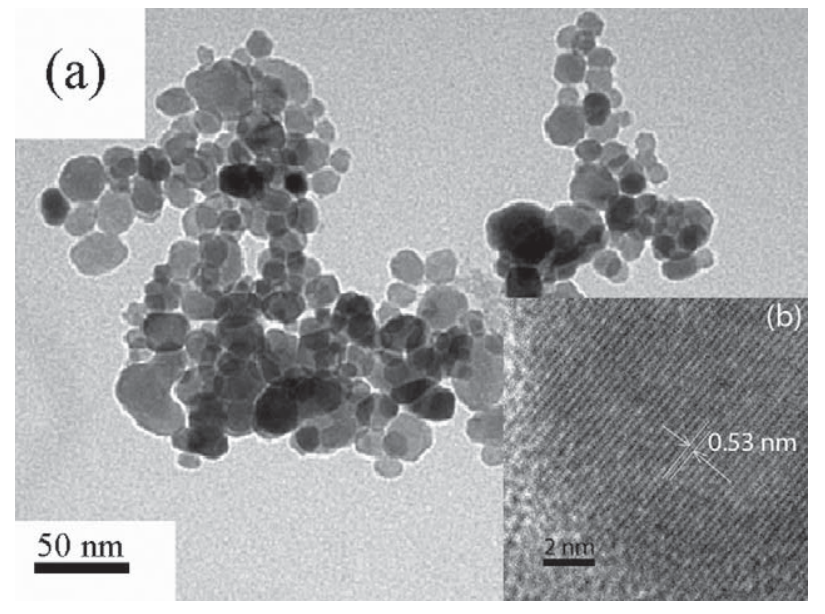

Fig. 1. TEM images of 5 at\% Co-doped $\mathrm{ZnO}$ nanoparticles; (a) bright-field image, (b) high resolution lattice fringe image.

\subsection{Testing of photocatalysis}

The specific surface area of the particles was analysed by the Brunauer-Emmett-Teller (BET) gas absorption method using a Micromeritics Tristar 3000 system. All powders were dried at $150^{\circ} \mathrm{C}$ for $1 \mathrm{~h}$ under high purity nitrogen gas prior to analysis.

Rhodamine B (RhB) dye was used to evaluate the photocatalytic activity of doped and undoped $\mathrm{ZnO}$ nanoparticles in response to simulated sun light irradiation which includes UV and visible light. The optical absorption peak characteristic of $\mathrm{RhB}$ at $554 \mathrm{~nm}$ was chosen to monitor the dye degradation. The experiment was carried out according to the following procedure: $8 \mathrm{mg}$ of the dried powder was dispersed in $50 \mathrm{ml}$ of $\mathrm{RhB}$ aqueous solution having the concentration of $0.0096 \mathrm{~g} \mathrm{~L}^{-1}$ in a $50 \mathrm{ml}$ quartz beaker. The suspension was stirred in the dark for $1 \mathrm{~h}$ to ensure the establishment of adsorption and desorption equilibrium of RhB on the particle surface. Subsequently the suspension was irradiated with simulated sunlight using an Atlas Suntest CPS+ instrument equipped with a $150 \mathrm{~W}$ Xenon lamp. After every $30 \mathrm{~min}$ of irradiation, $3 \mathrm{ml}$ of the suspension was extracted and then centrifuged to separate particles from supernatant. The UV-Vis absorbance spectra of the supernatant were measured using a Varian Cary 3E spectrophotometer using a quartz cuvette having an optical path length of $10 \mathrm{~mm}$.

\section{Results and discussion}

\subsection{Morphology and structural analysis}

The colour of synthesized $\mathrm{ZnO}$ nanopowders varied from pure white, light green to dark green as the Co composition increased from 0 to 5 at\%, consistent with the incorporation of a Co into the tetrahedral Zn site. Fig. 1(a) shows a typical bright field image of 5 at\% Co-doped $\mathrm{ZnO}$. It is evident that the powder consisted of agglomerated nanoparticles with the sizes between $10 \mathrm{~nm}$ and $50 \mathrm{~nm}$. The undoped $\mathrm{ZnO}$ and other Co-doped samples with different doping levels appeared nearly identical under TEM. Fig. 1(b) shows a high resolution TEM image of a doped $\mathrm{ZnO}$ nanoparticle, in which the measured interplanar spacing $(0.53 \mathrm{~nm})$ matches well with the value of a hexagonal wurtzite lattice parameters $(c=5.21 \AA)$.

Fig. 2 shows the correlation between the real dopant levels identified by ICP-AES and theoretical doping level. The real doping levels were close to the theoretical values and had a good liner correlation 


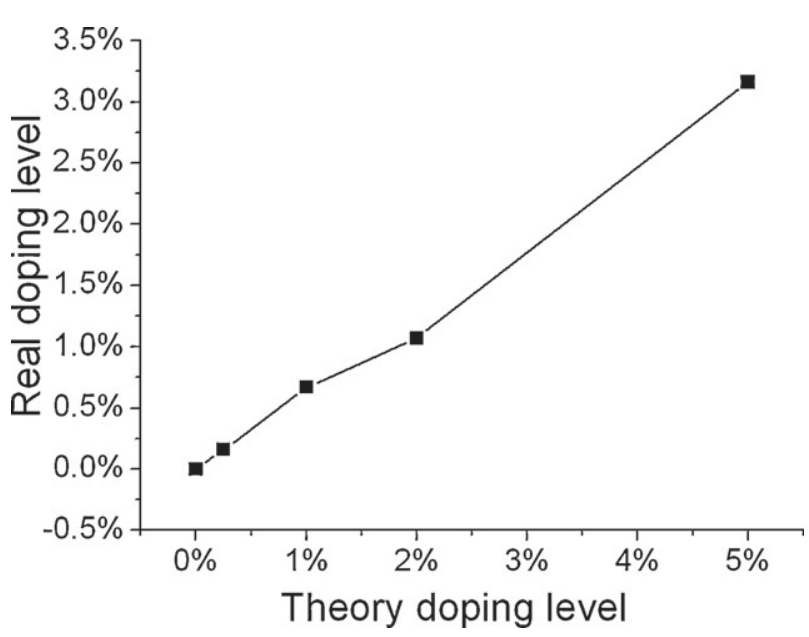

Fig. 2. Correlation between nominal and experimental Co doping levels.

with the nominal values. Hereafter we use nominal dopant levels to identify the samples in the text.

Zinc and cobalt have ionic radius of $0.60 \AA$ and $0.58 \AA$, respectively [21]. Hence, if Co replaces $\mathrm{Zn}$ in the $\mathrm{ZnO}$ crystal lattice, a reduction in lattice parameters is expected. A high flux X-ray source from synchrotron was considered to be useful to detect the small change in lattice parameters. Fig. 3(a) shows the XRD spectra of undoped and Co-doped ZnO samples. The XRD patterns of
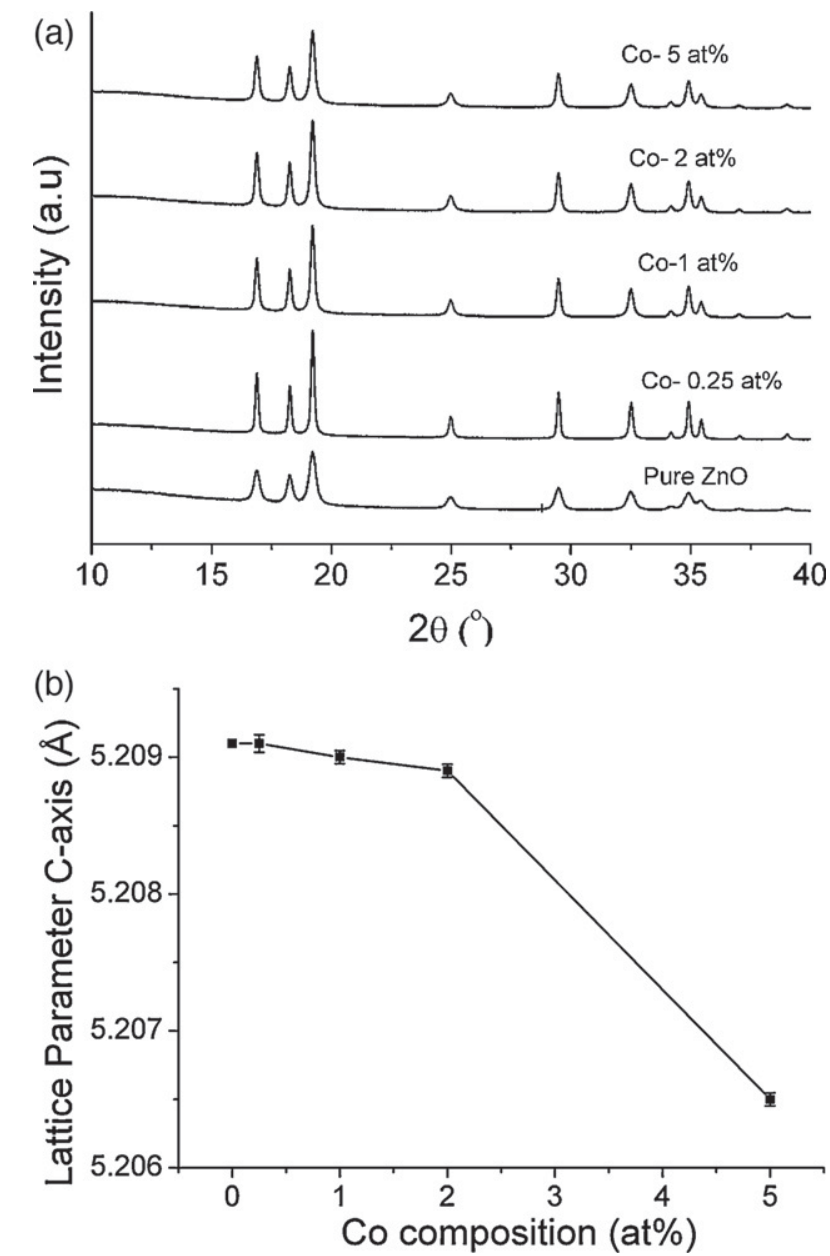

Fig. 3. (a) Synchrotron XRD spectra of undoped $\mathrm{ZnO}$ and Co-doped $\mathrm{ZnO}$ samples $(0.25,1,2$ and 5 at\%), (b) $c$-axis lattice parameter calculated by Retivielt analysis.

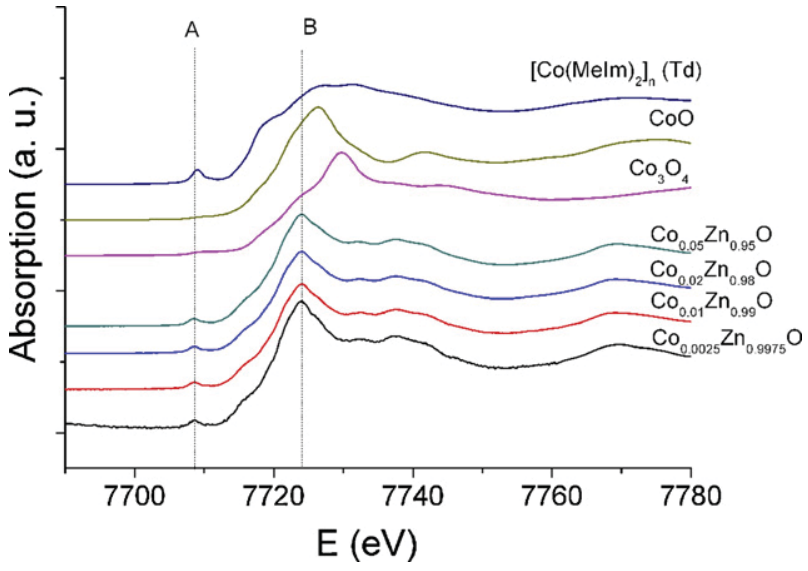

Fig. 4. K-edge XANES spectra for Co-doped ZnO and reference samples.

Co-doped $\mathrm{ZnO}$ samples showed the same characteristics as undoped $\mathrm{ZnO}$, consisting of only peaks corresponding to $\mathrm{ZnO}$. The peaks associated with separated cobalt oxide phases such as $\mathrm{Co}_{3} \mathrm{O}_{4}$ were not detected. Simple observation of diffraction-peak positions was not sufficient to detect the change in lattice parameters. Hence, the Rietveld analysis method [22-25] was employed to calculate the $c$-axis lattice parameter. As shown in Fig. 3(b), the $c$-axis lattice parameter decreased from $5.2091 \AA$ to $5.2065 \AA$ as the dopant level increased from 0 to 5 at\%, indicative of Co-atoms replacing zinc atoms in the $\mathrm{ZnO}$ lattice [26].

In addition to the powder diffraction data, XAS was carried out to investigate the location of the Co within the $\mathrm{ZnO}$ crystal lattice. There are three possibilities in the location: (1) The Co decorates the surface of the $\mathrm{ZnO}$ with a phase such as $\mathrm{CoO}$ or $\mathrm{Co}_{3} \mathrm{O}_{4}$, (2) the Co creates interstitial defects in the $\mathrm{ZnO}$ crystal lattice, or (3) the Co occupies $\mathrm{Zn}$ sites within the lattice. There are two parts of the XANES spectra that can be used to examine the conditions of doped Co ions, which are marked as pre-edge peak " $A$ " and rising edge " $\mathrm{B}$ " in Fig. 3 . The pre-edge peak $\mathrm{A}$ has been assigned as a $1 \mathrm{~s} \rightarrow 3 \mathrm{~d}$ transition $[27,28]$ and is therefore characteristic of all open-shell transition metals, so will be present in the $\mathrm{Co}(\mathrm{II})$, $3 d^{7}$, K-edge spectra. The intensity of the peak A is reflective of the coordination environment; a tetrahedral cobalt compound will have higher intensity than an octahedral compound [24,25]. This effect can be seen in Fig. 4 where the tetrahedral (Td) reference (Co-methylimidazole) has substantially higher peak $\mathrm{A}$ than $\mathrm{CoO}$ in which $\mathrm{Co}$ is present in octahedral $(\mathrm{Oh})$ sites. The high intensity of peak $\mathrm{A}$ in the spectra of Co-doped $\mathrm{ZnO}$ indicates that the majority of doped Co-ions were present in tetrahedral sites.

The rising edge energy $\mathrm{B}$ is indicative of oxidation states. The rising edge energy position of Co-doped $\mathrm{ZnO}$ are similar to each other and to that of the $\mathrm{Td}$ reference and $\mathrm{CoO}$ reference, indicating that the oxidation state of most of the Co were +2 . The above observations of XANES spectra indicate that Co ions substituted $\mathrm{Zn}$ sites in the wurtzite crystal lattice.

In addition to the XANES data, interpretation of EXAFS can also be used to elucidate the location of the Co ions. Fig. 5(a) shows $k^{3}$ weighted Co K-edge EXAFS spectra and Fig. 4(b) shows the scaled phase-corrected Fourier Transforms, for the series of Co-doped ZnO samples. They are compared with the Zn K-edge EXAFS and its Fourier Transform of undoped ZnO. The Fourier Transforms of EXAFS represent the local atomic structure around the atom of interest. The position and height of the peaks in Fourier Transforms are associated with the distance and number of neighbouring atoms. For example, in the Fourier Transforms of Zn K-edge EXAFS of undoped $\mathrm{ZnO}$, the first peak at $1.9 \AA$ corresponds to the first coordination shell with 4 oxygen atoms. The second peak at $3.3 \AA$ 

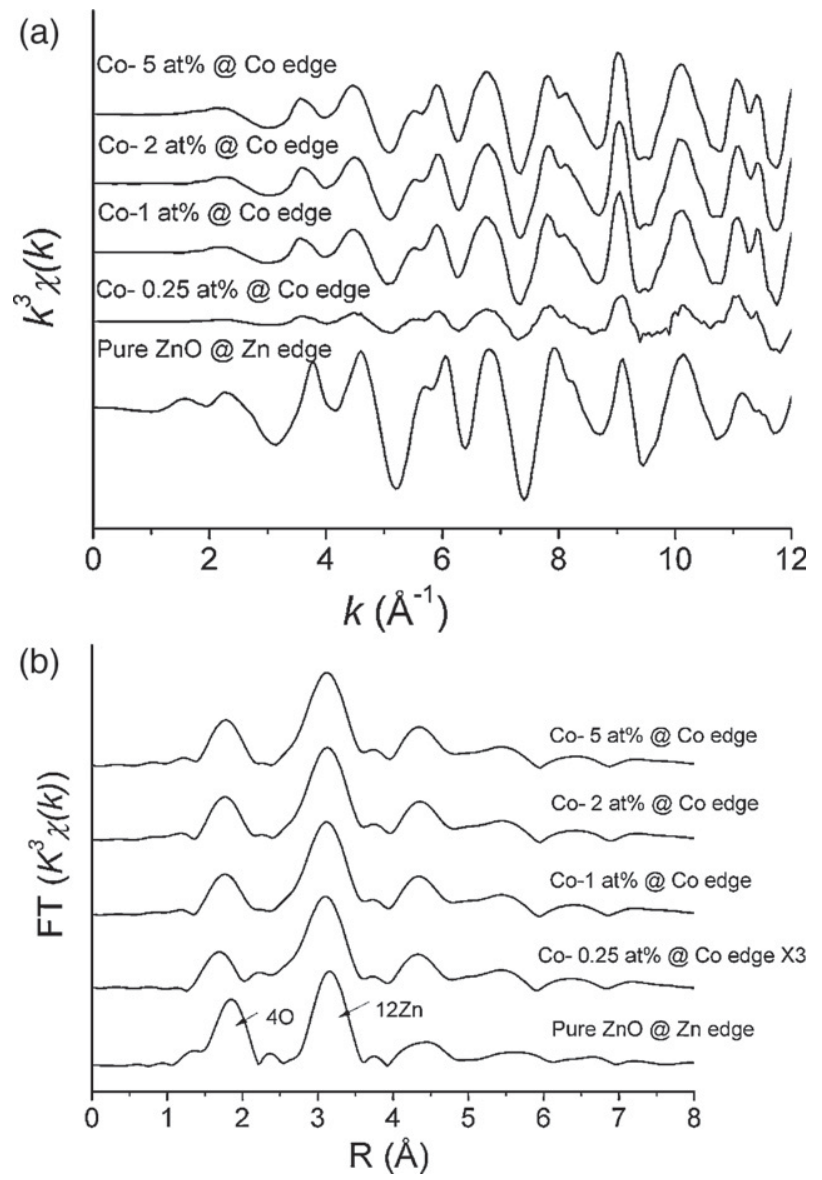

Fig. 5. (a) Co K-edge EXAFS $k^{3} \chi(k)$ functions for Co-doped $\mathrm{ZnO}$ and the Zn K-edge function for un-doped $\mathrm{ZnO}$, (b) Fourier Transforms of the EXAFS spectra of un-doped and Co-doped ZnO.

represents the second coordination sphere, consisting of $12 \mathrm{Zn}$ atoms at $3.3 \AA$. The Fourier Transforms of Co K-edge EXAFS represent the distance and number of neighbouring atoms around $\mathrm{Co}$ dopants.

As shown in Fig. 5(a), the EXAFS patterns across the series of Co-doped ZnO samples are nearly identical to each other in oscillation shape but not in intensity. The low intensity of the EXAFS pattern of 0.25 at\% doped $\mathrm{ZnO}$ is caused by self-absorption phenomena [29]. Since $\mathrm{Zn}$ and Co have similar atomic numbers, when the doping level is very low, materials cannot be diluted with BN powder adequately for XAS measurements to minimise self absorption [30]. The possibility of reduced EXAFS intensity by the presence of another crystal phase or disordered structures can be ruled out, because uniform reduction of the intensity across the entire EXAFS range was observed which is not expected to occur by the presence of another crystal phase or disordered structure. Hence, it is reasonable to scale the intensity of Fourier Transforms for comparison without causing any distortion in the data interpretation. In Fig. 5(b), the intensity of the Fourier Transforms of 0.25 at\% Codoped $\mathrm{ZnO}$ was magnified 3 time so that the peak heights become similar to those in the Fourier Transforms of other samples.

It is evident that the shape of Fourier Transforms for the Co-K-edge EXAFS of doped $\mathrm{ZnO}$ is nearly identical to that for the $\mathrm{Zn}$-K-edge EXAFS of undoped $\mathrm{ZnO}$. The result indicate that the local atomic arrangement around doped Co ions were almost the same as that around $\mathrm{Zn}$ ions, suggesting that the doped Co atoms substituted the $\mathrm{Zn}$ atoms in the $\mathrm{ZnO}$ crystal lattice [31,32], which supports the results of both the XANES and XRD analysis described above.
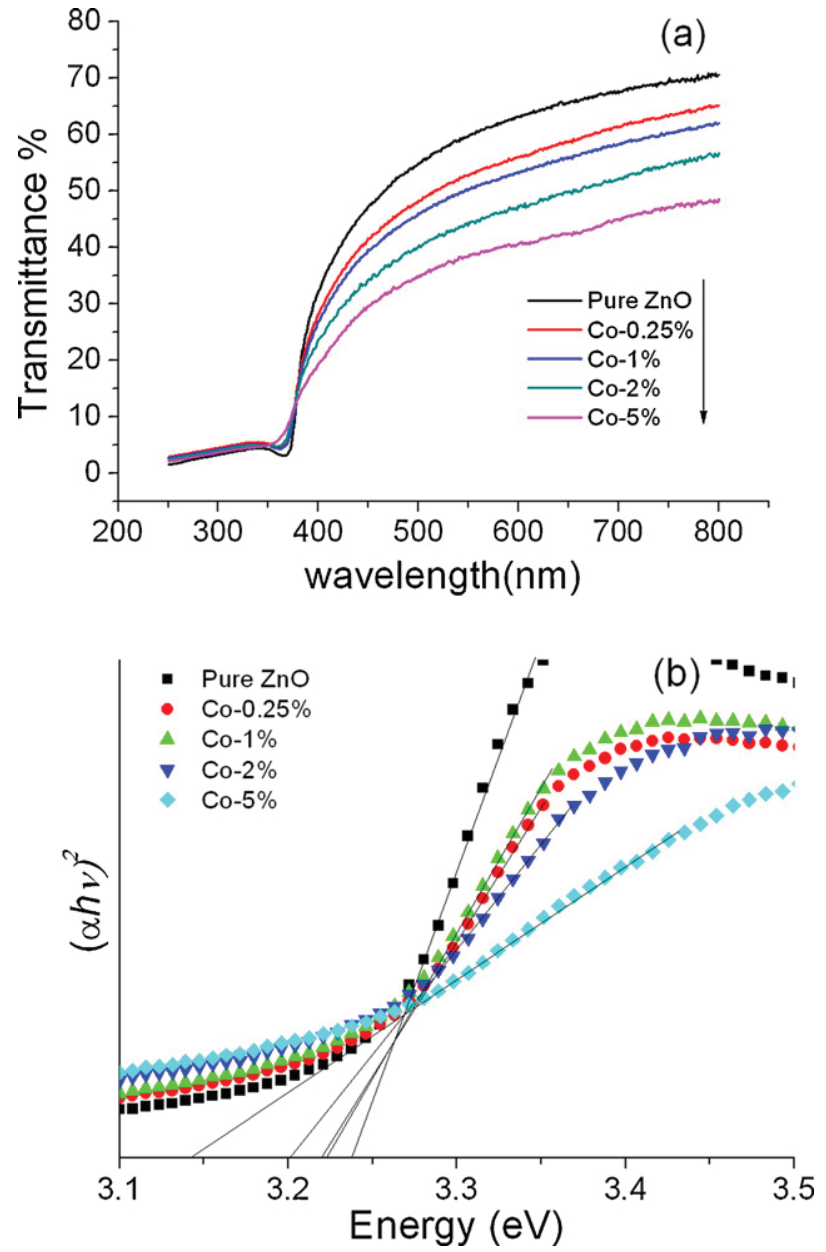

Fig. 6. (a) UV-Vis transmittance spectra and (b) estimation of band gap energies of undoped and Co-doped $\mathrm{ZnO}$.

\subsection{Photocatalytic property}

The UV-Vis transmittance spectra of undoped and doped $\mathrm{ZnO}$ are shown in Fig. 6. It is evident that the increased doping level resulted in a decrease in optical transmittance in the visible light range, while retaining the good absorption property in the UV-light range. The bandgap energy of the samples were also calculated by extrapolating the linear portion of the plot $(\alpha h v)^{2}$ versus $h v$ to the $(\alpha h v)^{2}=0$ axis [33,34]. The estimated bandgap energy of Co-doped $\mathrm{ZnO}$ gradually decreased from the value of pure $\mathrm{ZnO}(3.24 \mathrm{eV})$, which is similar to the earlier red-shift observation $[35,36]$. This red shift phenomenon is mainly due to the $s p-d$ exchange interactions between the localized $d$ electrons and the band electrons of the $\mathrm{Co}^{2+}$ ions which substitute $\mathrm{Zn}$ ions [37].

Fig. 7 shows the doping effects on the BET specific surface area. Although there is a significant variation in the trend as a function of doping level, the surface area values did not differ much among the samples.

Fig. 8(a) shows the relative change in the intensity of the optical absorption peak of RhB dye as a function of irradiation time. In the $y$ axis of Fig. 8(a), $C$ is the absorbance value of $\mathrm{RhB}$ at each irradiation time interval at the wavelength of $554 \mathrm{~nm}$ and $C_{0}$ is the absorbance value before the irradiation when the adsorption and desorption equilibrium was achieved. The data was normalised using the BET specific surface area of each sample. It is evident that the photoinduced degradation of Rhodamine-B was significantly slower in the presence of doped $\mathrm{ZnO}$ than undoped $\mathrm{ZnO}$. Higher doping levels resulted in stronger reduction of Rhodamine-B decomposition. 


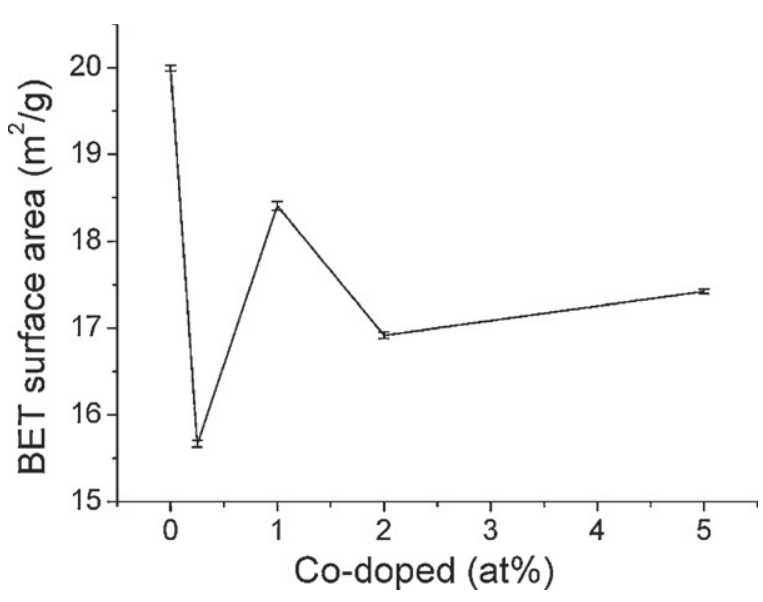

Fig. 7. BET surface areas of undoped and Co-doped $\mathrm{ZnO}$.

Fig. 8(b) shows the calculated Rhodamine-B degradation rate assuming the first-order kinetics [38]. It is evident that doping level of 0.25 at\% was sufficient to cause a meaningful decrease in the photo-degradation rate. Doping of 1 at\% induced a significant decrease in the photo-degradation rate down to $18 \%$ of the original value. Above the doping level of 1 at\%, the photo-degradation showed only slight decrease as the doping level increased. The photos of the Rhodamine- $\mathrm{B}$ solutions with un-doped $\mathrm{ZnO}$ under $1.5 \mathrm{~h}$ irradiation and with 5 at\% Co-doped $\mathrm{ZnO}$ under $2 \mathrm{~h}$ irradiation are
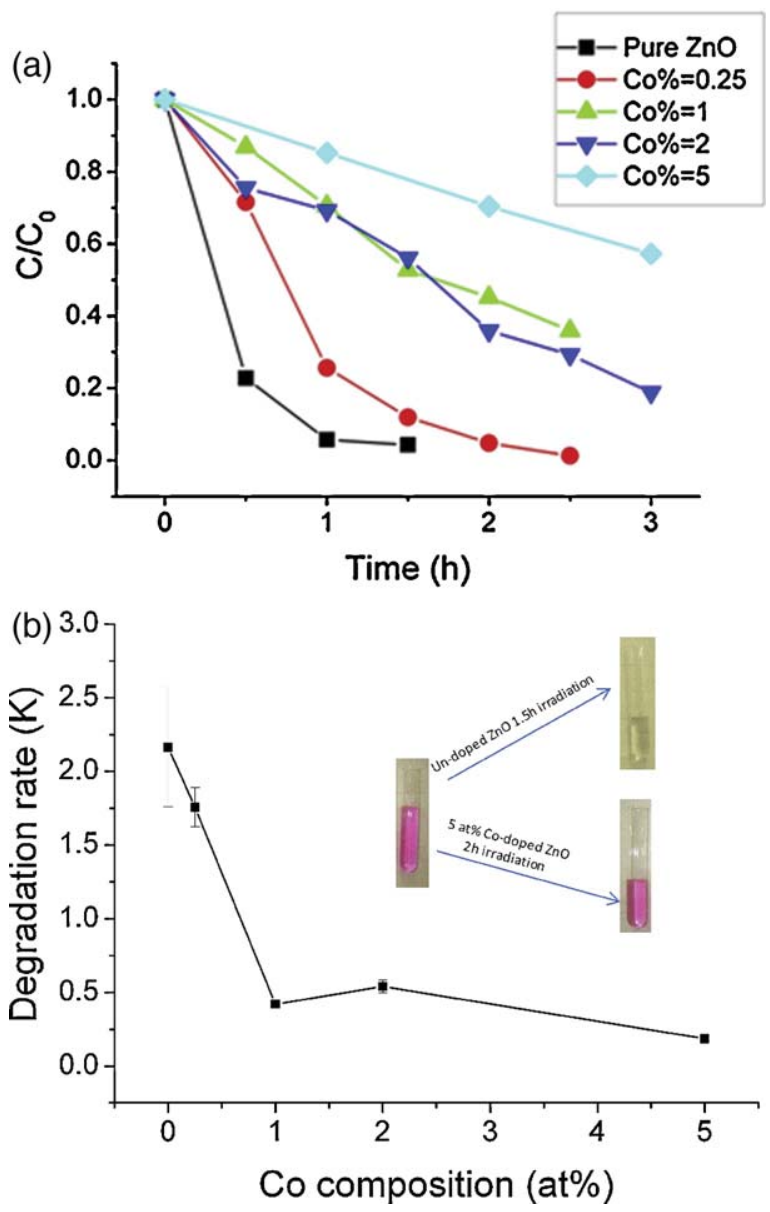

Fig. 8. (a) Relative change in the concentration of Rhodamine-B molecules as a function of light-irradiation time in the presence of un-doped and Co-doped $\mathrm{ZnO}$, (b) calculated Rhodamine-B degradation rates. also shown in Fig. 8(b), illustrating the strong effect of Co-doping on the decrease of the photocatalytic property in $\mathrm{ZnO}$.

The successful lattice doping of Co in $\mathrm{ZnO}$ may lie in the fact that the ionic radii of $\mathrm{Co}$ and $\mathrm{Zn}$ are close to each other. Co-doping did not appear to create significant physical defects in $\mathrm{ZnO}$ crystal. Hence the mechanism of the reduction of the photocatalytic activity by Co-doping is considered to be not by creating physical defects but mainly by introducing deep band gap energy levels between the valence and conduction bands that would act as efficient recombination centers for photo-generated excitons [39,40]. The recombination of electrons and holes inside of the particles will give the charge carriers less chance to generate ${ }^{\bullet} \mathrm{OH}$ and $\mathrm{O}_{2}{ }^{-}$free radicals on particle surfaces [13].

\section{Conclusions}

In this study, the location of dopant ions and the effect of doping level on the photocatalytic activity under the simulated sunlight have been investigated on Co-doped $\mathrm{ZnO}$ nanopowders. A sol-gel co-precipitation method and subsequent heat treatment were used to synthesize Co-doped $\mathrm{ZnO}$ nano-powders of up to 5 at\% doping level. Synchrotron X-ray diffraction and X-ray absorption spectra were used along with TEM study to show that Co atoms successfully replaced the $\mathrm{Zn}$ atoms in the $\mathrm{ZnO}$ lattice. This may have created deep band gap energy levels for the recombination of photo-generated electrons and holes. It was demonstrated that Codoping can effectively decrease the photocatalytic activity of $\mathrm{ZnO}$ and that the photo-degradation rate can be controlled by varying Co-doping levels. Only 1 at\% of Co-doping reduced the photoactivity down to $18 \%$ of the original value. Although Co ions are toxic, encapsulation of $\mathrm{Co}$-doped $\mathrm{ZnO}$ in a hydrophobic environment may facilitate the practical use of the material as a durable UV shielding agent for the protection of plastics, textiles and other organic matters from UV rays.

\section{Acknowledgements}

The authors acknowledge kind assistance from Prof. Terry Turney, Dr. Chris Glover, and Dr. Justin Kimpton on EXAFS and XRD measurements at Australian Synchrotron and Dr. Deanna D'Alessandro for allowing us to use her $\mathrm{T}_{\mathrm{d}}$ Co network compound as part of this study. Part of this research was undertaken on the Powder Diffraction and X-ray Absorption beamlines at the Australian Synchrotron, Victoria, Australia via proposals M1397 and M1503.

\section{References}

[1] H. Hosono, Thin Solid Films 515 (2007) 6000-6014.

[2] A. Becheri, M. Durr, P. Lo Nostro, P. Baglioni, J. Nanoparticle Res. 10 (2008) 679-689.

[3] D. Beydoun, R. Amal, G. Low, S. McEvoy, J. Nanoparticle Res. 1 (1999) 439-458.

[4] T. Tsuzuki, J.S. Robinson, P.G. McCormick, J. Aust. Ceram. Soc. 38 (2002) 15-19.

[5] P.J. Barker, A. Branch, Prog. Org. Coat. 62 (2008) 313-320.

[6] S.R. Morrison, T. Freund, J. Chem. Phys. 47 (1967) 1543-1546.

[7] B. Bems, F.C. Jentoft, R. Schlogl, Appl. Catal., B: Environ. 20 (1999) 155-163.

[8] M. Lackhoff, X. Prieto, N. Nestle, F. Dehn, R. Niessner, Appl. Catal., B: Environ. 43 (2003) 205-216.

[9] N. Serpone, A. Salinaro, A. Emeline, Nanoparticles Nanostruct. Surf.: Novel Rep. Biol. Appl. 2 (2001) 86-98.

[10] A. Dodd, A. McKinley, T. Tsuzuki, M. Saunders, Mater. Chem. Phys. 114 (2009) 382-386.

[11] J.P. Han, P.Q. Mantas, A.M.R. Senos, J. Eur. Ceram. Soc. 22 (2002) 49-59.

[12] T.F. Jaramillo, S.-H. Baeck, A. Kleiman-Shwarsctein, K.-S. Choi, G.D. Stucky, E.W. McFarland, J. Comb. Chem. 7 (2004) 264-271.

[13] S. Ekambaram, Y. Iikubo, A. Kudo, J. Alloy Compd. 433 (2007) 237-240.

[14] P.S. Casey, C.J. Rossouw, S. Boskovic, K.A. Lawrence, T.W. Turney, Superlattice Microstruct. 39 (2006) 97-106.

[15] J.M.D. Coey, M. Venkatesan, C.B. Fitzgerald, Nat. Mater. 4 (2005) 173-179.

[16] H.J. Lee, S.H. Choi, C.R. Cho, H.K. Kim, S.Y. Jeong, Europhys. Lett. 72 (2005) 76-82.

[17] B. Hunter, Int. Union Crystallogr. Comm. Powder Diffract. Newslett. 20 (1998) $18-24$. 
[18] C. Glover, J. McKinlay, M. Clift, B. Carg, J. Boldman, M. Ridgeway, G. Foran, R. Garrett, P. Lay, A. Broadbent, AIP Conference Proceedings (2007) 882 (X-Ray Absorption Fine Structure (XAFS13)), 2007, pp. 884-886.

19] Australian National Beam-line Facility and the Australian Synchrotron, 2008-2010. Average http://www.synchrotron.org.au/index.php/ aussyncbeamlines/x-ray-absorption-spectroscopy/data-analysis.

[20] B. Ravel, M. Newville, J. Synchrotron. Radiat. 12 (2005) 537-541.

[21] S. Chang, V.V. Karambelkar, R.D. Sommer, A.L. Rheingold, D.P. Goldberg, Inorg. Chem. 41 (2001) 239-248.

[22] W.I.F. David, J. Appl. Crystallogr. 37 (2004) 621-628.

[23] R.J. Hill, C.J. Howard, J. Appl. Crystallogr. 20 (1987) 467-474.

24] D.B. Wiles, R.A. Young, J. Appl. Crystallogr. 14 (1981) 149-151.

$25]$ K. Shankland, J. Res. Natl. Inst. Stan. 109 (2004) 143-154.

[26] X.C. Liu, E.W. Shi, Z.Z. Chen, H.W. Zhang, B. Xiao, L.X. Song, Appl. Phys. Lett. 88 (2006) 252501-252503

[27] T.E. Westre, P. Kennepohl, J.G. DeWitt, B. Hedman, K.O. Hodgson, E.I. Solomon, J. Am. Chem. Soc. 119 (1997) 6297-6314

28] J.E. Hahn, R.A. Scott, K.O. Hodgson, S. Doniach, S.R. Desjardins, E.I. Solomon, Chem. Phys. Lett. 88 (1982) 585-588.
[29] J. Osán, F. Meirer, V. Groma, S. Török, D. Ingerle, C. Streli, G. Pepponi, Spectrochim. Acta B: Atom. Spectrosc. 65 (2010) 1008-1013.

[30] I.J. Pickering, G.N. George, E.Y. Yu, D.C. Brune, C. Tuschak, J. Overmann, J.T. Beatty, R.C. Prince, Biochemistry: US 40 (2001) 8138-8145.

[31] V. Ischenko, S. Polarz, D. Grote, V. Stavarache, K. Fink, M. Driess, Adv. Funct. Mater. 15 (2005) 1945-1954.

[32] X.C. Liu, E.W. Shi, Z.Z. Chen, B.Y. Chen, W. Huang, L.X. Song, K.J. Zhou, M.Q. Cui Z. Xie, B. He, S.Q. Wei, J. Alloy Compd. 463 (2008) 435-439.

[33] U. Koch, A. Fojtik, H. Weller, A. Henglein, Chem. Phys. Lett. 122 (1985) 507-510.

[34] L. Brus, J. Phys. Chem. 90 (1986) 2555-2560.

[35] S.V. Bhat, F.L. Deepak, Solid State Commun. 135 (2005) 345-347.

[36] K.J. Kim, Y.R. Park, Appl. Phys. Lett. 81 (2002) 1420-1422.

37] Y.R. Lee, A.K. Ramdas, R.L. Aggarwal, Phys. Rev. B 38 (1988) 10600-10610.

[38] J. Wang, T. Tsuzuki, B. Tang, P. Cizek, L. Sun, X. Wang, Colloid Polym. Sci. 288 (2010) 1705-1711.

[39] J. Han, P.Q. Mantas, A.M.R. Senos, J. Eur. Ceram. Soc. 22 (2002) 49-59.

[40] M. Jakani, G. Campet, J. Claverie, D. Fichou, J. Pouliquen, J. Kossanyi, J. Solid State Chem. 56 (1985) 269-277. 